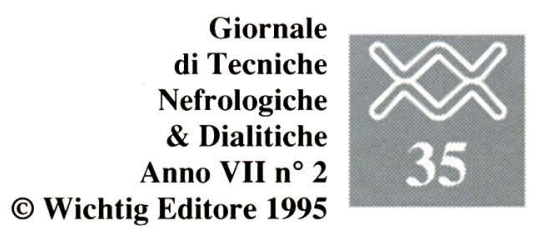

\title{
Diagnostica ecografica delle nefropatie mediche
}

\author{
P. Conti
}

\author{
Servizio di Nefrologia USL 10 - Sorgono (NU)
}

ecotomografia si è ormai affermata in campo nefrologico per lo studio morfologico dei reni. Il nefrologo, senza nulla togliere a chi da sempre si è interessato della diagnostica per immagini, si è impossessato di questa metodica riuscendo a coniugare l'immagine con i dati clinici e di laboratorio in suo possesso. Pertanto in campo nefrologico, così povero di semeiologia fisica, questo strumento permette di organizzare e favorire il ragionamento clinico.

Tutto questo senza dimenticare che l'ecotomografia rappresenta un'indagine strumentale che obbligatoriamente deve integrarsi con quelle più tradizionali $\mathrm{e}$ più sofisticate (che restano di assoluta pertinanza della radiologia), anche se queste ultime spesso per la loro natura necessitano di tempi lunghi per la loro ripetizione. Questo non è necessario in ecografia, che permette di seguire, in tempo reale ed in certe patologie, l'evoluzione clinica della malattia, effettuando esami ripetuti, con intervalli di tempo molto ridotti.

La pratica ecografica permetterà poi di valutare in maniera più ampia la patologie dell'apparato urinario, con lo studio della vescica e, nell'uomo, della prostata. L'ecografia renale, comunque, difficilmente potrà condurre ad una diagnosi di natura delle patologie renali, ma permetterà certamente lo studio delle caratteristiche del parenchima renale, e que- sto favorendo sempre un orientamento diagnostico. In altre parole non potremo mai fare una diagnosi definitiva di nefropatia medica, ma certamente sarà possibile per esempio l'evidenziazione della flogosi del parenchima renale che, inquadrandola in un preciso contesto clinico, ci sarà di grande aiuto nella probabile diagnosi; inoltre sarà possibile lo studio dell'evoluzione della malattia (1-5).

In ecografia potremo studiare la grandezza dei reni, la loro simmetria, la regolarità del profilo, lo spessore e l'ecogenicità del parenchima funzionante, la grandezza e l'ecogenicità del seno pielico, certe caratteristiche del peduncolo va- scolare; ancora l'ecografia ci permette di ricercare eventuali immagini anomale iperecogene (per esempio calcoli) o ipoecogene (per esempio cisti) o lesioni o masse presenti a vari livelli del parenchima renale.

La valutazione delle dimensioni dei reni è forse la più semplice ed immediata. Nella tabella I sono elencate le cause più frequenti delle variazioni delle dimensioni e della simmetria renale.

Patologie renali acute, oppure anche non in fase di acuzie, possono non mostrare alcuna anomalia ecografica (Fig. 1). La nefropatia $\operatorname{IgA}$ può presentare reni apparentemente normali, anche se il paziente
Figura 1 - Ecografia sostanzialmente normale in un giovane paziente affetto da glomerulopatia di Berger: l'indagine ecografica è stata effettuata in corso di macroematuria.

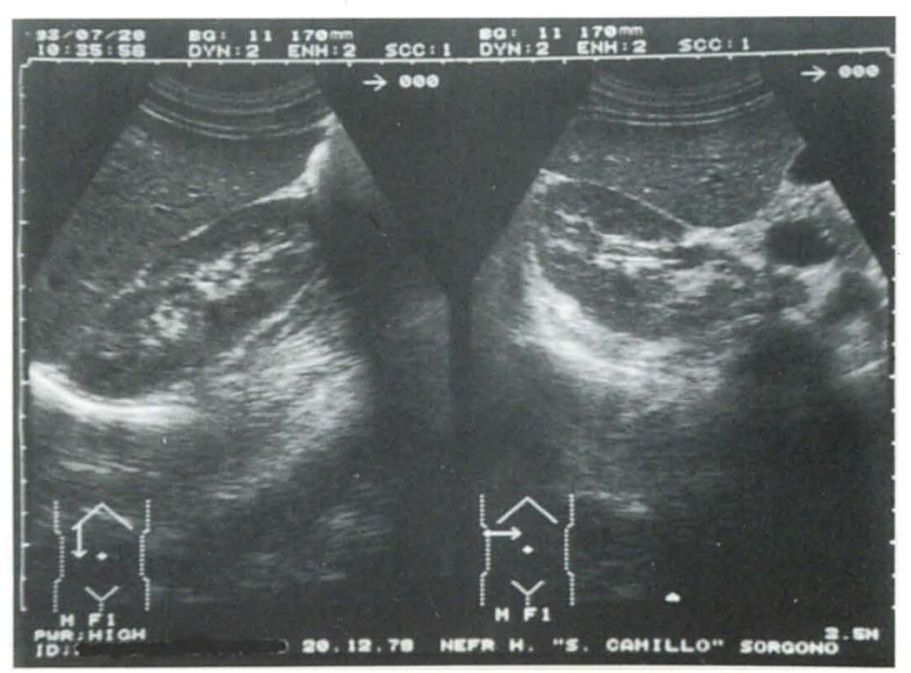


TAB. I - CAUSE PRINCIPALI DI ANOMALIE DELLE DIMENSIONI E DELLA SIMMETRIA DEI RENI

Reni aumentati (mono o bilateralmente):

a - edema

1) glomerulonefrite acuta

2) interstiziopatia acuta

b - accumulo ematico

1) trombosi vena renale

c - accumulo di lipidi

1) glomerulonefrite a lesioni minime

d - nefroni ipertrofici

e - cisti semplici

1) singola

2) multiple

f - reni policistici

$\mathrm{g}$ - tumore renale

Reni diminuiti simmetricamente:

a - nefropatie vascolari (inclusa nefrosclerosi benigna)

b - glomerulonefriti evolutive primitive

c - glomerulonefriti evolutive secondarie

$\mathrm{d}$ - glomerulonefrite cronica

e - reni end-stage

Reni diminuiti asimmetricamente:

a - pielonefrite cronica

b - infarti guariti

Reni aumentati o normali:

a - diabete mellito

b - amiloidosi

Diminuzione singola di un rene:

a - ostruzione della arteria renale

presenta macroematuria.

L'ecogenicità del parenchima renale funzionante ed il suo spessore sono alterati in numerose condizioni acute: un aumento di spessore del parenchima con diffusa e marcata ipoecogenicità, legata a fenomeni di edema, è reperto caratteristico delle glomerulonefriti acute sia primitive che secondarie (fig. 2), nelle quali si può osservare anche una riduzione del seno pelvico, dovuta alla compressione corticomidollare. Un reperto analogo (fig. 3) può essere osservato anche in condizioni di edema interstiziale a rapida insorgenza determinato da interstiziopatie che possono riconoscere varie cause (infettive, tossiche, da ipoperfusione renale, fino alla necrosi corticale, o anche conseguente a necrosi tubulare acuta): in questa condizione è particolarmente evidente la ipoecogenicità delle piramidi, e talora si osservano piccoli echi disseminati di debole intensità.
Flogosi localizzate (fig. 4) che in genere riconoscono una causa infettiva come nella pielonefrite focale, appaiono come zone solide ipoecogene localizzate a livello del parenchima funzionante, di difficile delimitazione, contenenti alcuni echi anche di notevole intensità. Tali aree possono essere talvolta intensamente ipoecogene e questo, se non sono presenti segni clinici rilevanti (dolore, febbre, piuria) può porre seri problemi di diagnosi differenziale con lesioni tipo cistiche, ascesso, tumore renale (fig. 5).

L'ascesso renale può essere comunque l'evoluzione liquefattiva di una flogosi focale. È anch'esso in genere caratterizzato da un'area ipoecogena, talora mal delimitata oppure limitata da una struttura pseudocapsulare. Nel contesto ipoecogeno si possono reperire alcuni echi di numero ed intensità variabile e spesso è presente un rinforzo ecografico posteriore. Quando l'ascesso è costituito da note- vole cellularità o sono presenti numerosi detriti, l'area ascessuale raggiungerà una ecogenicità molto intensa.

Nella tubercolosi renale potremo avere aree come descritte per l'ascesso renale (fig. 6, fig. 7), ma qui concomitano in genere aree anecogene espressione di lesioni cavitarie, oppure di idrocalici fino all'idronefrosi che può insorgere per stenosi ureterale: frequenti poi sono le calcificazioni, caratteristiche della nefropatia tubercolare.

Le micosi renali si esprimono ecograficamente in maniera molto varia, con masse iso o iperecogene nel contesto del parenchima renale.

Di maggiore interesse, anche perchè oggi rappresentano una grossa percentuale dei pazienti che seguiamo nei nostri ambulatori, sono le alterazioni croniche del parenchima.

Fenomeni di fibrosi si esprimono con un aumento dell'ecogenicità del parenchima renale. Questi possono essere ben localizzati, talvolta "a raggiera" (fig. 8), spesso accompagnati da incisure presenti sul profilo renale (esiti di tubercolosi renale, guarigione di infarti renali, cicatrici da flogosi nella pielonefrite focale) o diffusi: quest'ultimo caso è caratteristico nelle evoluzioni croniche delle glomerulonefriti (fig. 9), nelle pielonefriti croniche (fig. 10, fig. 11) e nelle nefropatie nei pazienti vasculopatici (fig. 12); se il danno cronico si accompagna ad una progressiva riduzione della funzione renale, in genere determinata da una riduzione della massa nefronica, si osserverà una diminuzione dello spessore della corticale renale ed anche della midollare, con tendenza all'espansione, effettiva o relativa, del seno pielico con la cosidetta "corticalizzazione dei calici".

La nefropatia potrà evolvere fino al quadro del rene grinzo, o rene end-stage: $i$ reni sono piccoli con marcato e diffuso aumento dell'ecogenicità fino alla scomparsa della differenziazione tra corticale, midollare e seno pelvico (fig. 13). È questo il reperto più comune nel paziente uremico terminale che viene avviato al trattamento depurativo sostitutivo.

Molto si è discusso e si discute sul rapporto tra riduzione della clearance renale e riduzione dello spessore del parenchima funzionante: riteniamo interessante questa disquisizione, ma nella nostra pratica non facciamo mai solo riferimento allo spessore della corticale per valutare la funzione renale. 


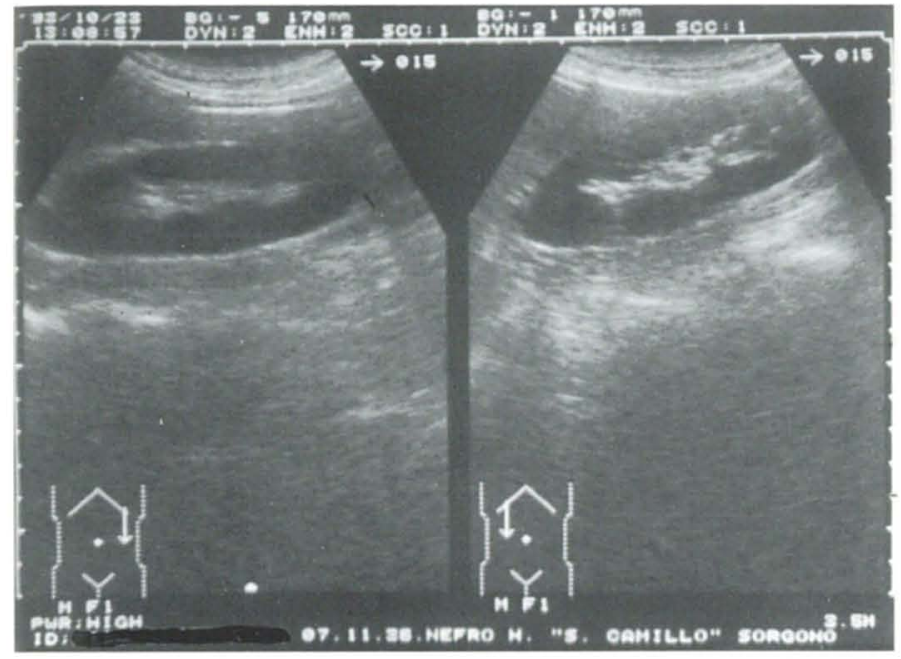

Figura 2 - Glomerulonefrite post-streptococcica: aumento di spessore del parenchima funzionante che appare ipoecogeno e riduzione del seno pelvico.

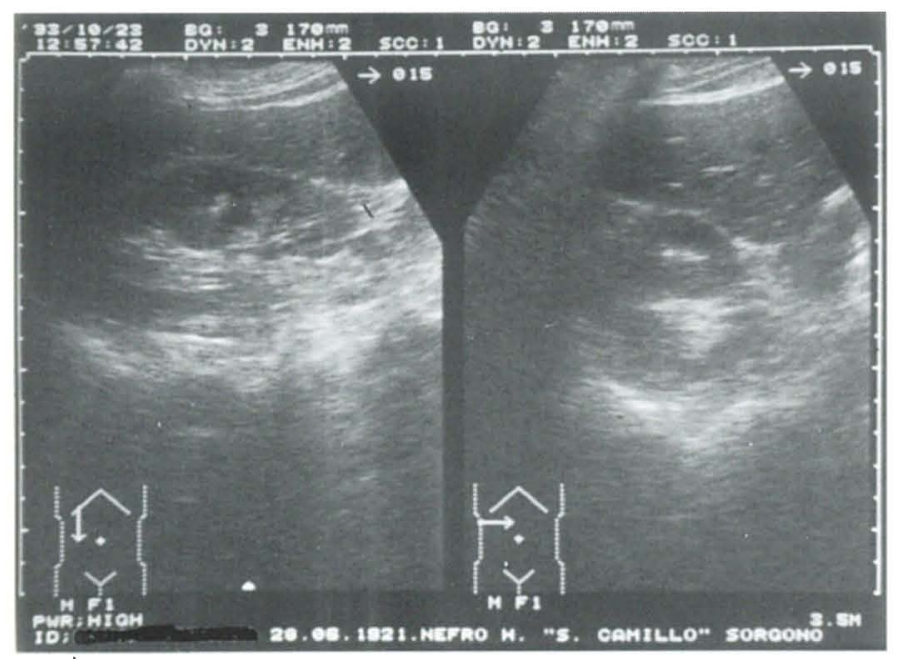

Figura 4 - Alterazione localizzata nel parenchima funzionante comprimente il seno pielico, di tipo sostanzialmente ipoecogeno, ma con intensi echi nel suo contesto, in paziente febbrile: nefropatia interstiziale focale.

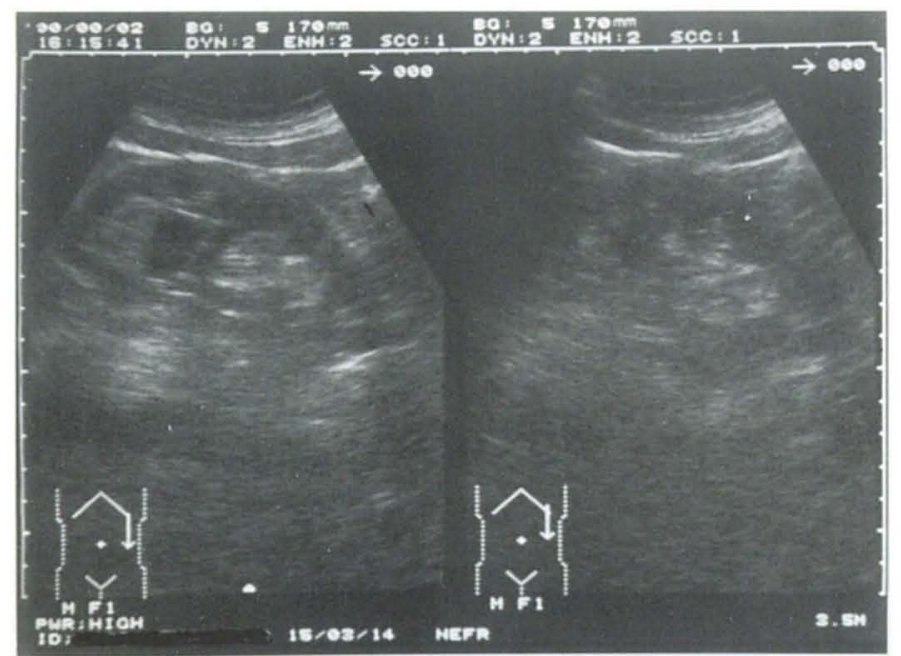

Figura 6 - Rene di dimensioni leggermente ridotte, con riduzione dello spessore del parenchima funzionante, un'area cavitaria e numerose aree calcifiche nel parenchima: nefropatia tubercolare.

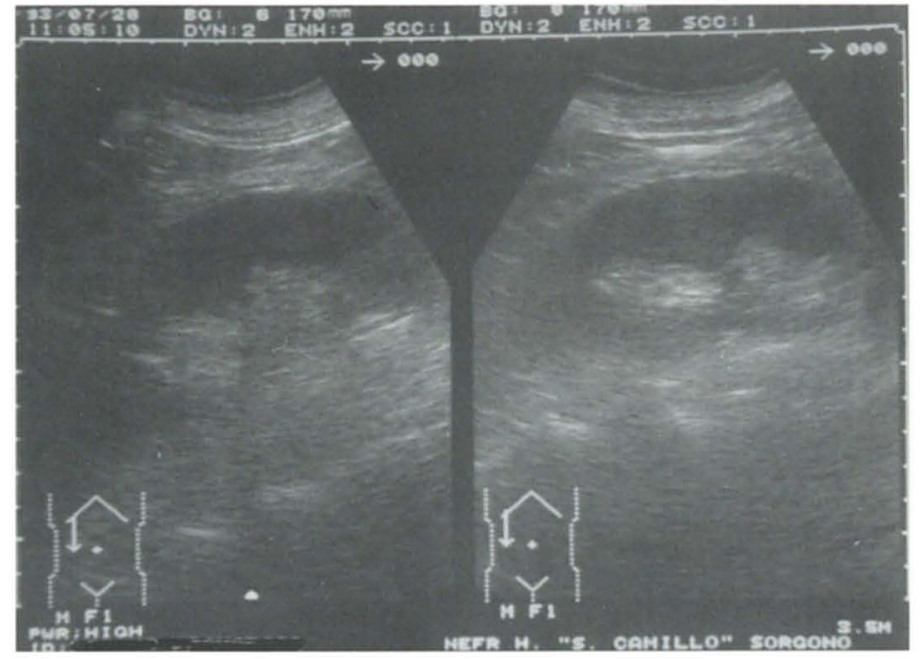

Figura 3 - Insufficienza renale acuta conseguente a shock in paziente con pancreatite: reni aumentati simmetricamente di volume con aumento dello spessore del parenchima funzionante.

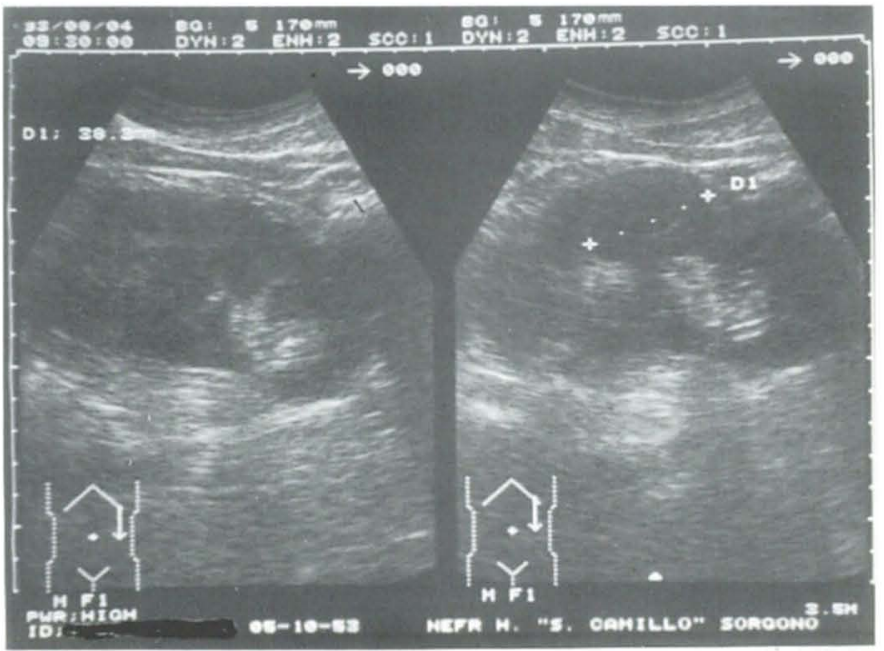

Figura 5 - Sfumata area ovalare ipoecogena, determinante una alterazione del profilo renale, non capsulata, come da raccolta ascessuale.

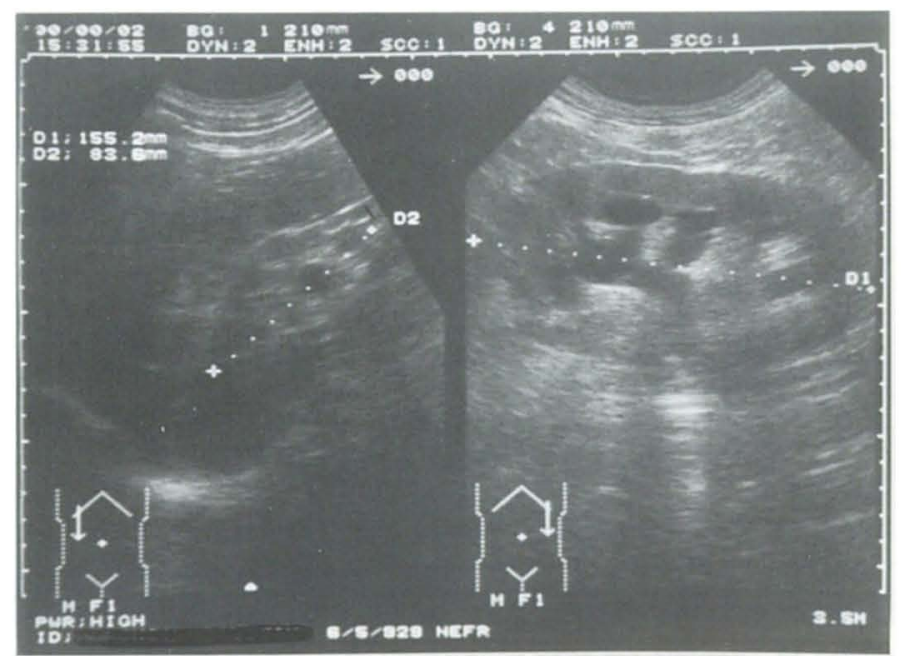

Figura 7 - Rene ds grinzo per nefropatia tubercolare; idronefrosi massiva sn da litiasi ureterale. Il paziente è giunto alla nostra osservazione per una IRA. 


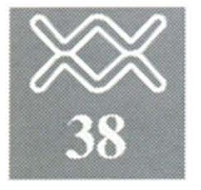

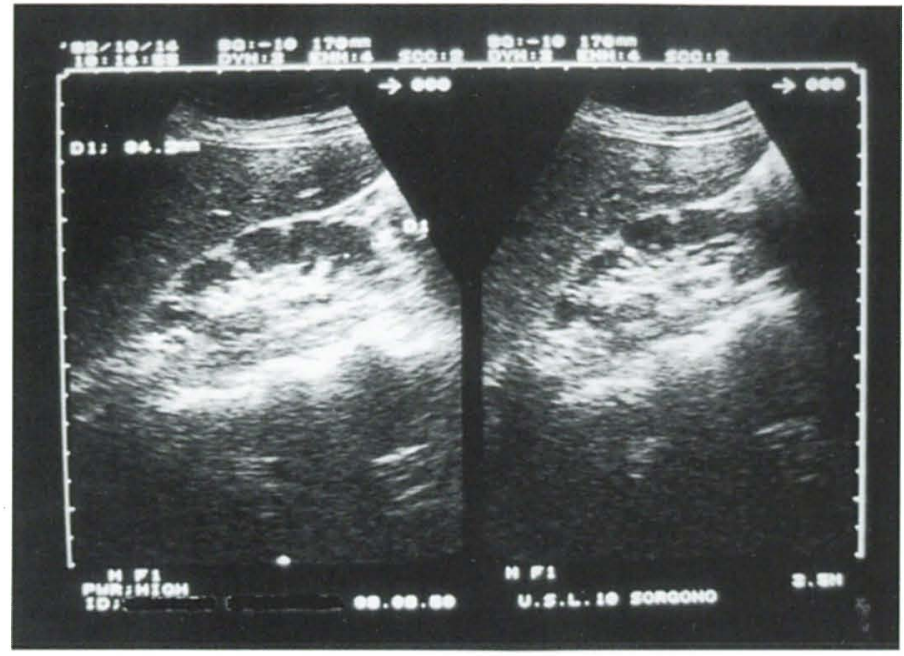

Figura 8 - Pielonefrite cronica. Rene di volume ridotto a profilo irregolare per la presenza di numerose incisure, con la corticale attraversata da immagini iperecogene, espressione di cicatrici del parenchima renale.

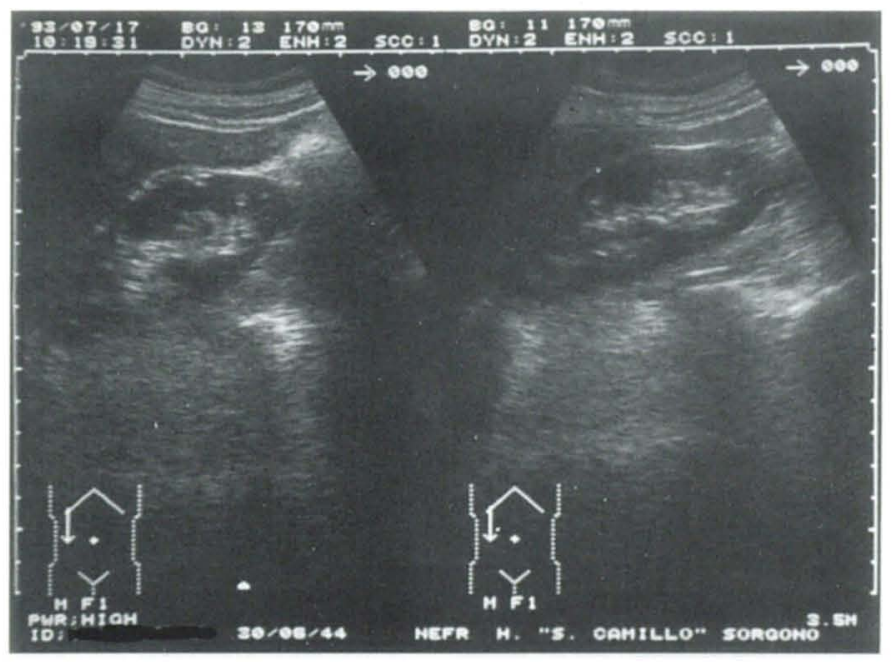

Figura 10 - Pielonefrite cronica; reni ridotti asimmetricamente: nel contesto del parenchima renale si osservano piccole immagini iperecogene da riferire a calcificazioni.

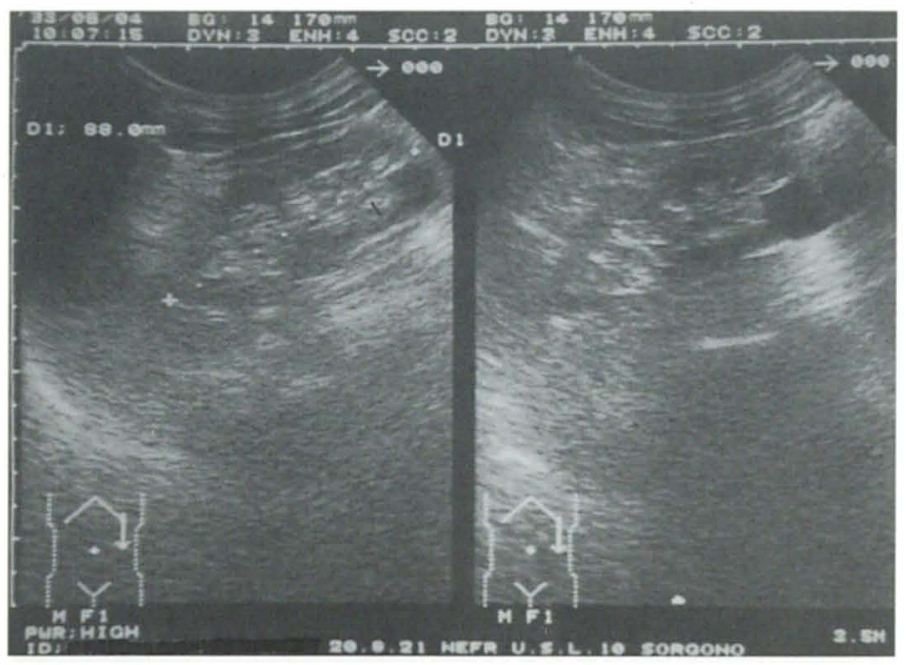

Figura 12 - Paziente con grave vasculopatia generalizzata ed IRC (crea-s 3,6 $\mathrm{mg} / \mathrm{dl}$ ); reni ridotti di grandezza con assottigliamento della corticale ed aumento diffuso dell' ecogenicità renale; piccola cisti al polo inferiore del rene sn.

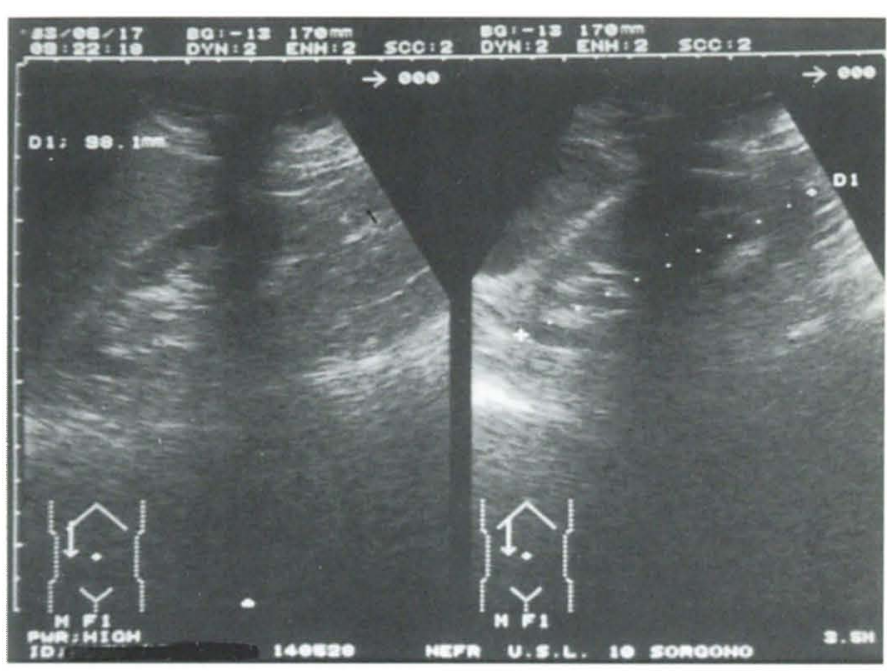

Figura 9 - Glomerulonefrite membrano-proliferativa in paziente con crioglobulinemia mista essenziale; modesta riduzione simmetrica della grandezza dei reni con assottigliamento del parenchima funzionante.



Figura 11 - Pielonefrite cronica; paziente monorene sn per nefrectomia ds da litiasi renale e ureterocutaneostomia sn: il rene superstite appare ipertrofico con importanti irregolarità dell' ecogenicità del parenchima, profilo irregolare (crea-s $1,7 \mathrm{mg} / \mathrm{dl}$ ).

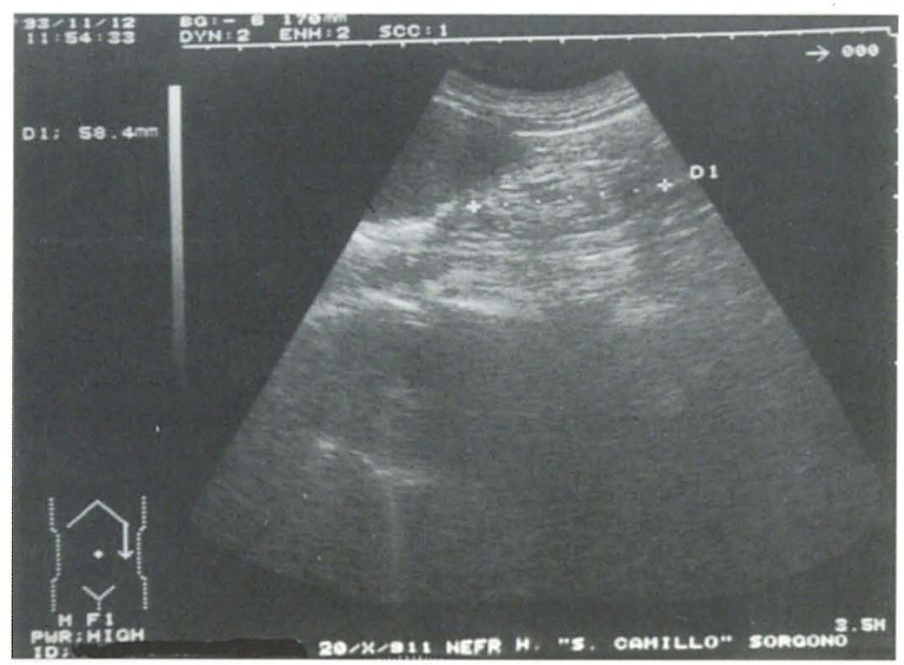

Figura 13 - Rene end-stage; di piccole dimensioni e scomparsa della normale ecostruttura del parenchima, che appare omogeneamente iperecogeno. 
L'assottigliamento del parenchima resta un dato importante che utilizziamo sempre nel controllo temporale del paziente con IRC, ma integrandolo con lo studio metabolico.

L'aumento dell'ecogenicità senza magari una particolare riduzione delle dimensioni dei reni potremo osservarla in varie patologie che possono interessare il parenchima renale. La condizione più ricordata è l'amiloidosi renale (6), dove l'accumulo di fibrille di amiloide si esprime con il marcato aumento dell'ecogenicità (fig. 14). Nella nostra esperienza però altre patologie hanno queste caratteristiche come per esempio l'artrite reumatoide, il LES, la nefropatia uratica e quella da crioglobulinemia (fig. 15).

Nel linfoma (7) potremo trovare una diminuzione dell'ecostruttura, che può interessare in varia misura il parenchima, con alternanza quindi di aree ipoecogene omogenee ad aree di aumentata ecogenicità, a margini non definiti (fig. 16). Un quadro analogo può essere riscontrato nel rene in pazienti affetti da leucemia (fig. 17).

Un cenno particolare meritano le espressioni ecografiche della nefropatia diabetica, la quale, d'altronde, ha varie espressioni cliniche. Nella nostra esperienza il quadro più frequente è rappresentato da reni bilateralmente aumentati di grandezza con riduzione del rapporto corticomidollare, aumento volumetrico del seno pielico ed una fine iperecogenicità in toto del parenchima, con profili regolari (fig. 18). Questo quadro, maggiormente frequente nel diabete mellito insulino-dipendente rispetto a quello tipo-II, è indipendente dalla capacità filtrante e depurativa dell'organo. Quando la nefropatia evolve verso la fase uremica della IRC, è possibile, ma non costante, una riduzione volumetrica renale (fig. 19) con le caratteristiche descritte precedentemente per la nefropatia cronica. Questo può dipendere dall'interessamento vascolare, che è una complicanza del diabete mellito. Ricordiamo poi come siano possibili alterazioni della struttura quando si verificano flogosi generalizzate, come già descritte, o anche localizzate solo ad una porzione di un rene. La necrosi papillare è in genere rappresentata da un modesto aumento del bacinetto con visualizzazione della cavità creata dalla necrosi.

Zone di aumentata ecogenicità del parenchima circondate da corticale indenne, originanti o meno coni d'ombra ecogra- fici, sono espressione caratteristica della nefrocalcinosi (fig. 20), che può essere accompagnata o meno da insufficienza renale. La presenza delle ombre acustiche è determinata dalla grandezza delle calcificazioni. Ricordiamo infatti che aree calcifiche di dimensioni inferiori ai $3 \mathrm{~mm}$ difficilmente danno origine a cono d'ombra posteriore.

In questo caso sarà molto utile integrare lo studio ecografico renale con quello della regione del collo (fig. 21) nella ricerca di una patologia paratiroidea in genere sostenuta da un adenoma paratiroideo. L'adenoma paratiroideo è anche da ricercare nei pazienti uremici, nei quali l'osteopatia è una delle patologie conseguenti al trattamento sostitutivo.

L'altra patologia (fig. 22) di grande interesse che si esprime con area calcifica che, se di grande dimensione determina il cono d'ombra, è la calcolosi renale (8.), patologia che noi riteniamo di interesse medico, e non solo chirurgico, per l'importanza che assume la prevenzione metabolica soprattutto per ridurre il numero di interventi chirurgici ed i trattamenti di litotrissia, quest'ultimi che rappresentano una spesa economica rilevante, anche se meno destruenti dell'intervento classico. Oltre a questo ricordiamo come la calcolosi urinaria possa determinare complicanze renali, acute e croniche, che facilmente possono condurre alla riduzione della funzione depurativa, la cui espressione più eclatante è l'idronefrosi.

Altra patologia di rilevanza medica di frequentissimo riscontro è la nefropatia cistica (9). L'immagine cistica classica è rappresentata da un'area a limiti netti, a pareti in genere fini e regolari, rotondeggiante od ovalare, di varie dimensioni, anecogena all'interno, con rinforzo ecografico posteriore dovuto all'assenza di attenuazione degli ultrasuoni nel mezzo liquido in rapporto ai tessuti circostanti (fig. 23). La patologia cistica semplice o multipla è descritta come asintomatica, ma secondo la nostra esperienza e da alcuni dati della letteratura (10-12), questa si può associare statisticamente a vari sintomi e patologie quali il dolore, l'ipertensione arteriosa ed è, inoltre, di frequente riscontro nella insufficienza renale cronica. Situazioni ecografiche che possono simulare una o più cisti renali sono descritte in tabella II. La patologia cistica è comunque di grande interesse nefrologico per la possibilità della evi- denziazione di Reni Policistici (fig. 24), i quali determinano una uremia terminale. In questo caso i reni sono bilateralmente aumentati di volume e la loro normale ecostruttura è completamente alterata dalla presenza di un enorme numero di cisti di varie dimensioni. Generalmente nella forma dominante le cisti alterano i profili renali, con aspetto bozzuto, che invece sono conservati nella forma recessiva, dove le cisti hanno dimensioni minori.

Altra patologia cistica (13.) che sta assumendo grande interesse in campo nefrologico è rappresentata dalla malattia cistica acquisita del dializzato (ACKD), per l'alta incidenza di degenerazioni neoplastiche di questa malattia (fig. 25): le caratteristiche cliniche di queste cisti non differiscono da quelle descritte per le forme semplici, ma un accurato controllo ecografico effettuato con regolarità, nel tempo, permetterà di individuare precocemente eventuali comparse di forme cistiche atipiche.

Di non facile esecuzione in ecografia classica è lo studio del peduncolo vascolare del rene, di più facile accesso all'eco-doppler (14.). Possibile è, comunque, l'evidenziazione di aneurismi o di calcificazioni dell'arteria renale. Stenosi dell'arteria renale conducono ad una riduzione armonica della grandezza e della morfologia del rene interessato dalla stenosi (fig. 26). Questo, come sappiamo, frequentemente si associa ad ipertensione arteriosa.

La patologia venosa di più frequente riscontro è la trombosi della vena renale: si associa ad edema renale con aumento di volume e perdita della differenziazione corticomidollare con espansione del seno pielico; l'ingorgo ematico potrà determinare un'iperecogenicità della corticale. Il trombo potrà essere messo in evidenza nel lume della vena, come imma-

\section{TAB. II - DIAGNOSI DIFFERENZIA- LE DELLA PATOLOGIA CISTICA}

1 - Calicectasia

2 - Idronefrosi

3 - Pionefrosi

4 - Cavità tubercolare

5 - Ascesso

6 - Diverticolo caliceale

7 - Tumore cistico

8 - Necrosi di tessuto tumorale 


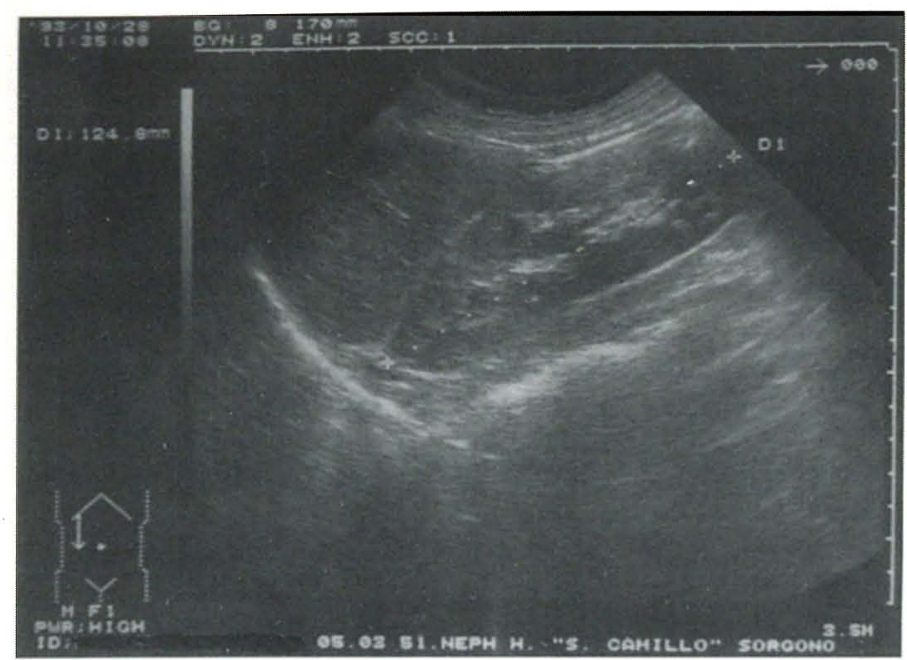

Figura 14 - Amiloidosi renale; tutto il parenchima appare aumentato di ecogenicità con notevole aumento del volume renale.

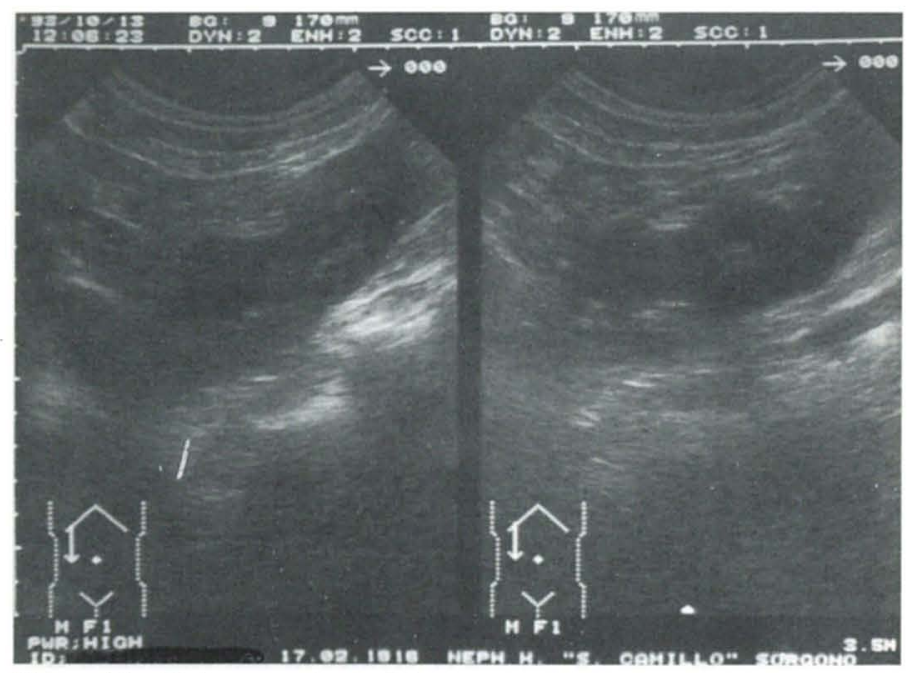

Figura 16 - Linfoma renale; la diffusa e marcata ipoecogenicità determina la completa scomparsa della normale ecostruttura del parenchima renale.

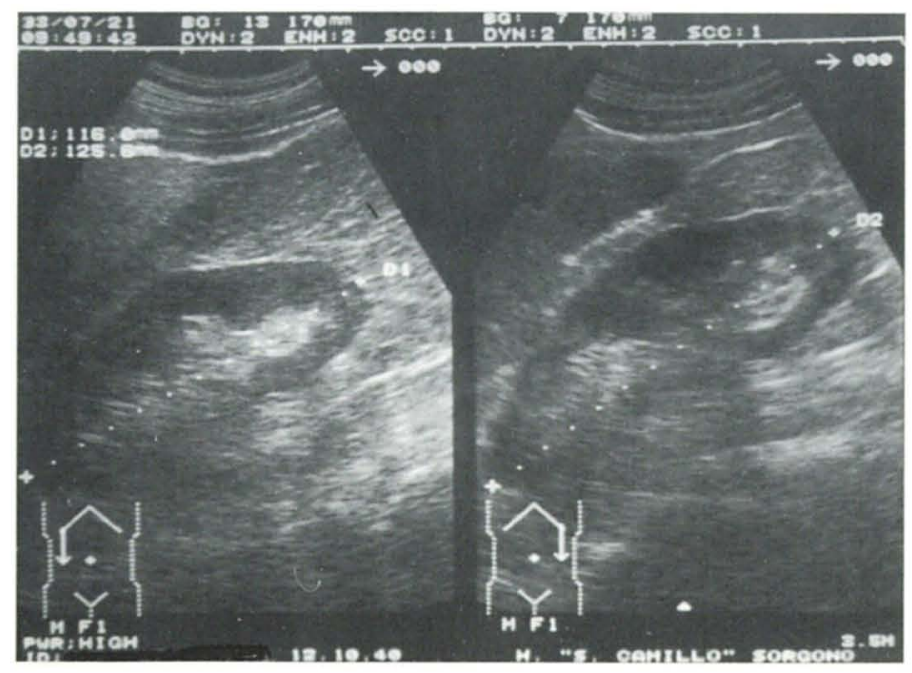

Figura 18 - Paziente affetto da diabete mellito tipo I; i reni sono nettamente aumentati di volume con ampiamento del seno pielico: aumento della trama ecostrutturale del parenchima.

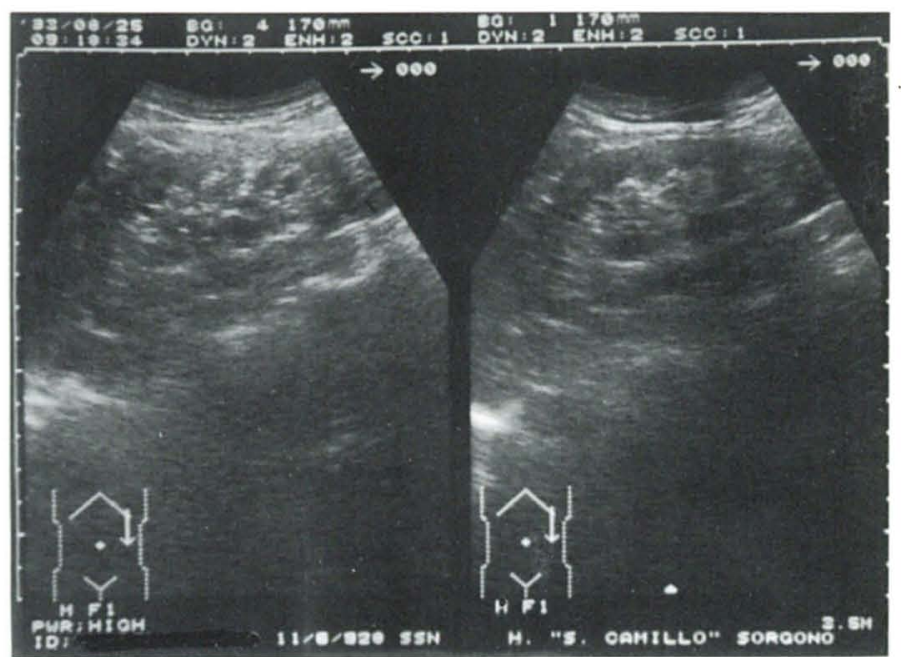

Figura 15 - Paziente affetta da artrite reumatoide; reni di dimensioni sostanzialmente normali, ma aumento in toto dell' ecogenicità del parenchima con riduzione dello spessore del parenchima funzionante (crea-s $1,1 \mathrm{mg} / \mathrm{dl}$; proteinuria $2,4 \mathrm{~g} /$ die)

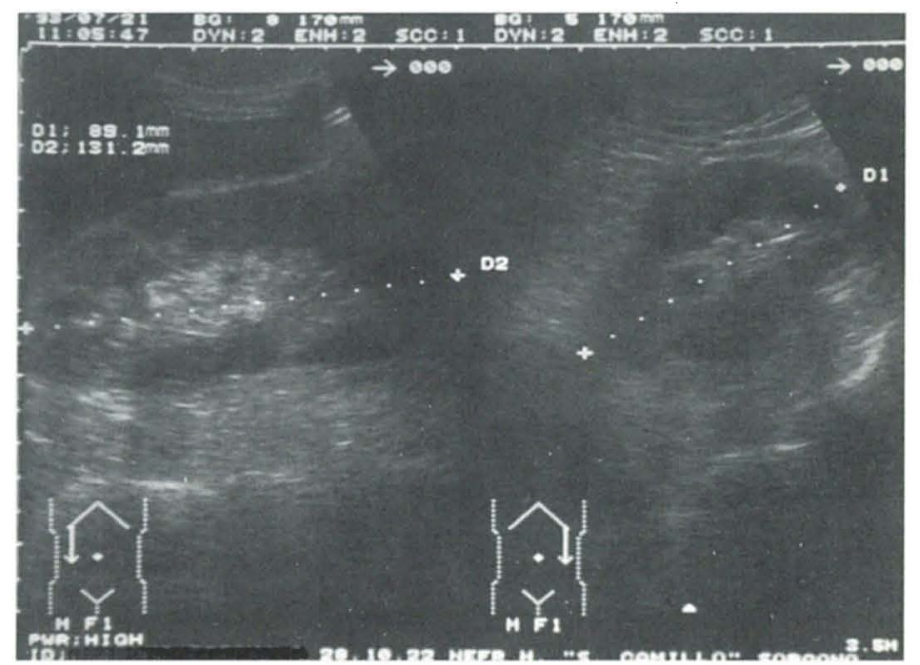

Figura 17 - Paziente con leucemia cronica; vi è asimmetria renale (con rene sn più piccolo con calcolo nel gruppo caliceale inferiore). Il parenchima mostra ampie zone di ipoecogenicità.

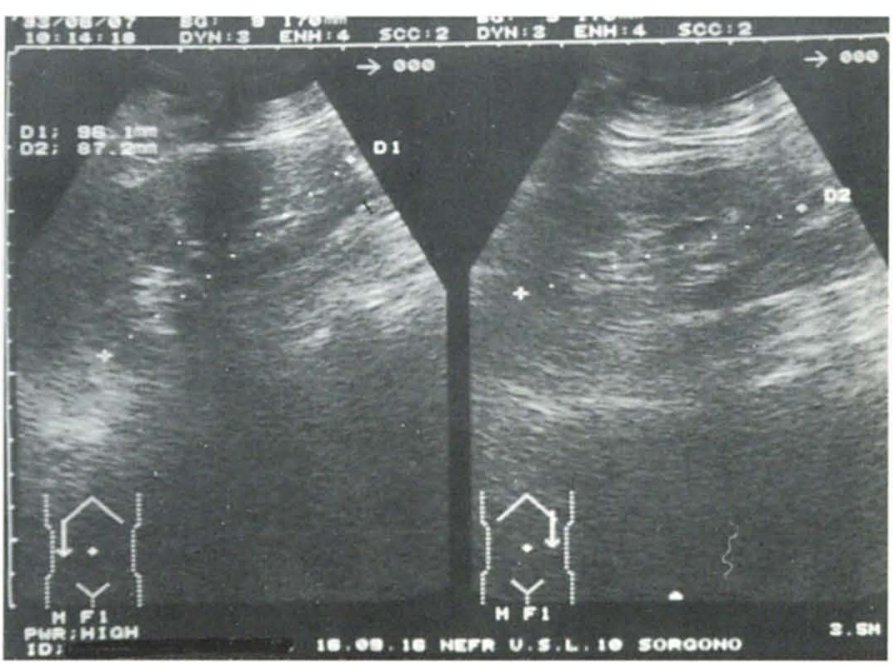

Figura 19 - Paziente con diabete mellito tipo I; reni ridotti di dimensioni, asimmetrici, marcato aumento dell' ecogenicità del parenchima (crea-s $1,6 \mathrm{mg} / \mathrm{dl}$ ). 


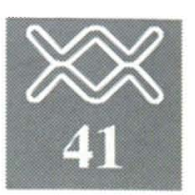

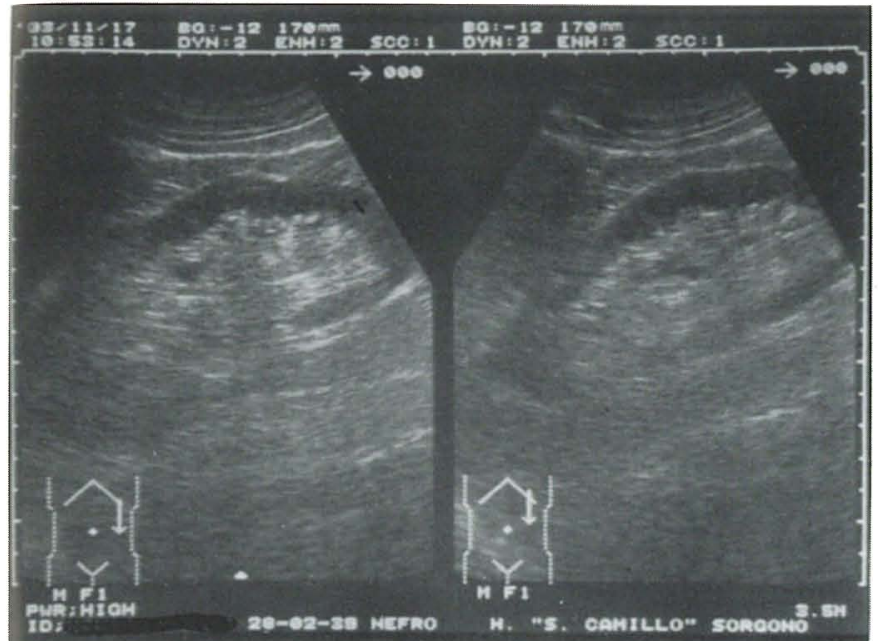

Fig. 20 - Nefrocalcinosi; numerose aree iperecogene localizzate nella midollare renale originanti coni d'ombra ecografici bilateralmente (calciuria 940 $\mathrm{mg} /$ die).

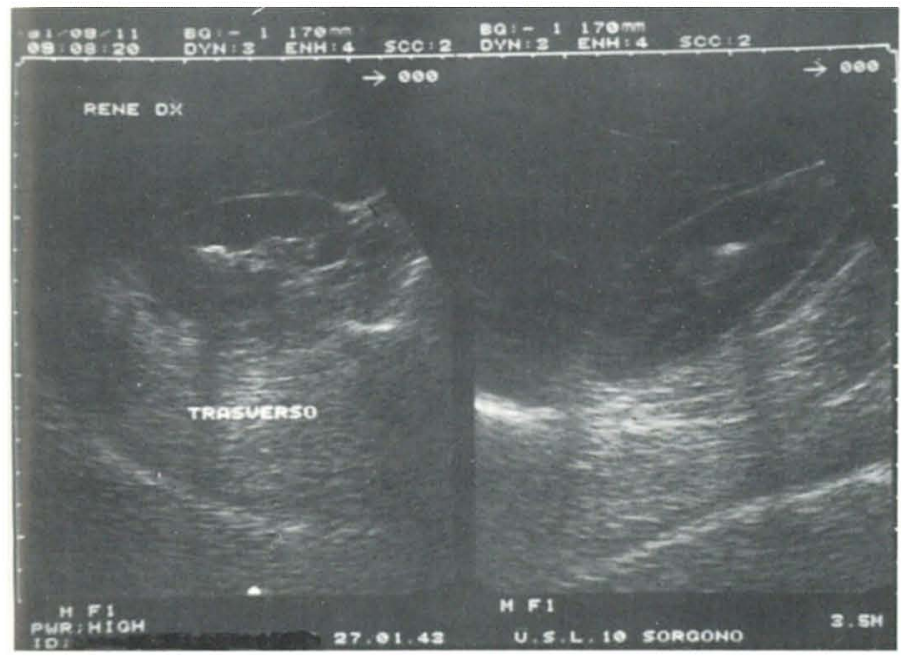

Figura 22: Calcolosi renale; grosso calcolo gruppo caliceale medio: si noti il marcatissimo cono d' ombra ecografico originante dall' area iperecogena.

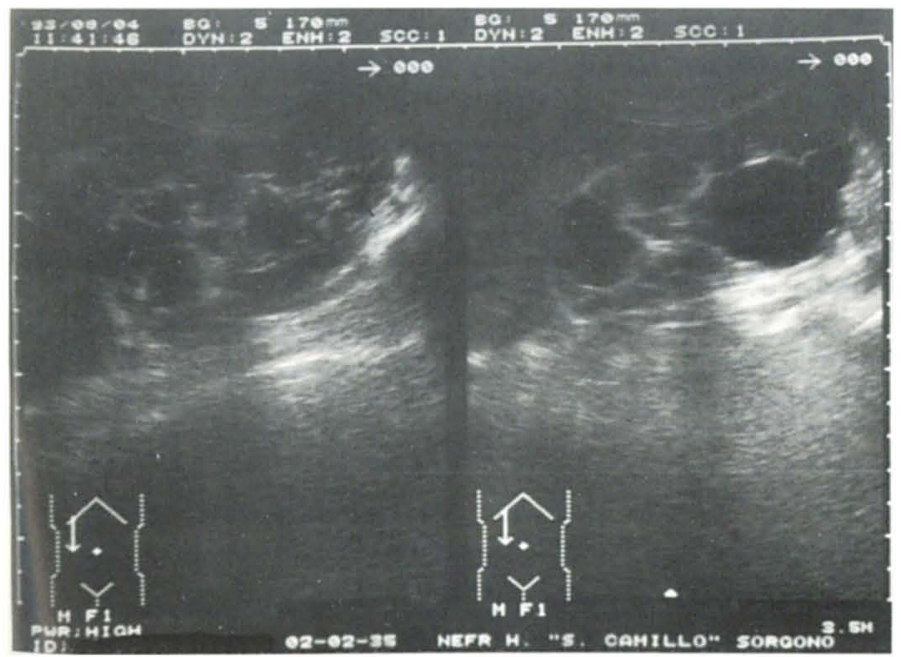

Figura 24: Patologia policistica dell' adulto (ADPKD); la morfologia renale è completamente alterata dalla presenza di un enorme numero di cisti di varie dimensioni che determinano un aumento volumetrico renale (paz. sottoposto a trattamento dialitico).

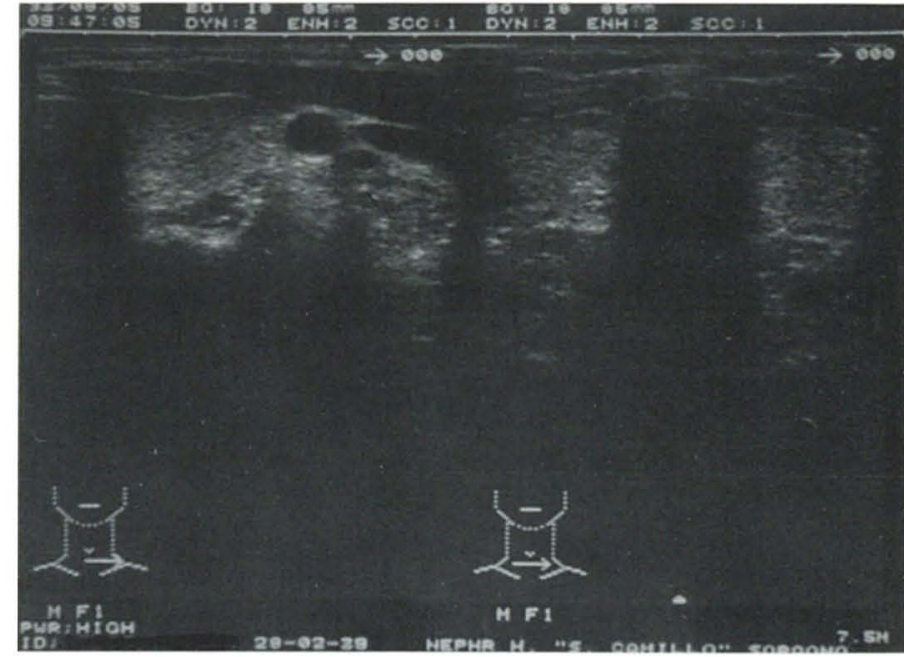

Figura 21: Ecografia della regione del collo; Area ipoecogena delle dimensioni di $6 \mathrm{~mm}$ localizzata posteriormente al lobo sn della tiroide: adenoma paratiroideo.

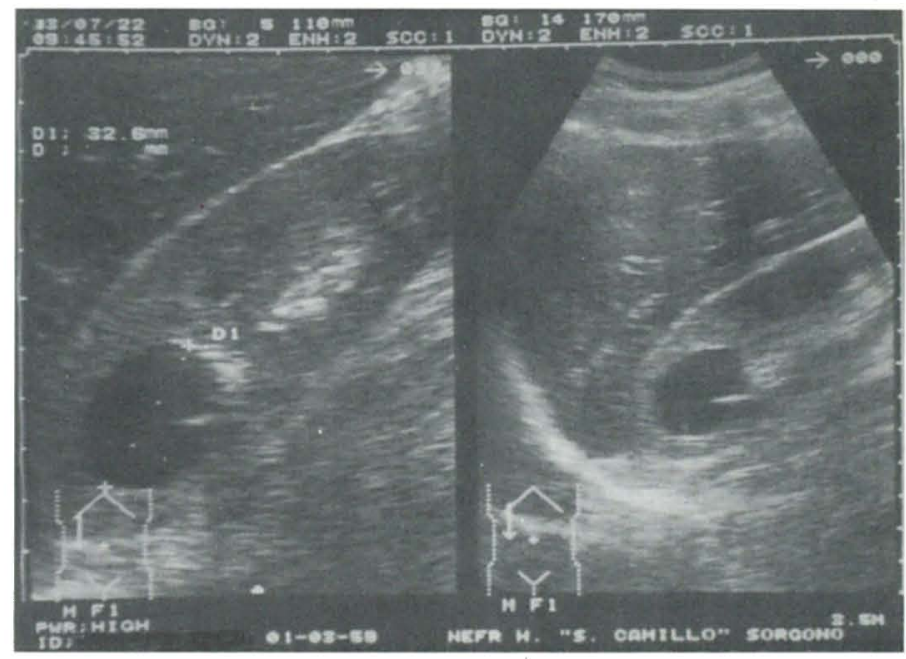

Figura 23: Cisti parapielica del polo superiore del rene ds: la cisti presenta, nel lume, un setto molto ben evidente all' indagine ecografica.

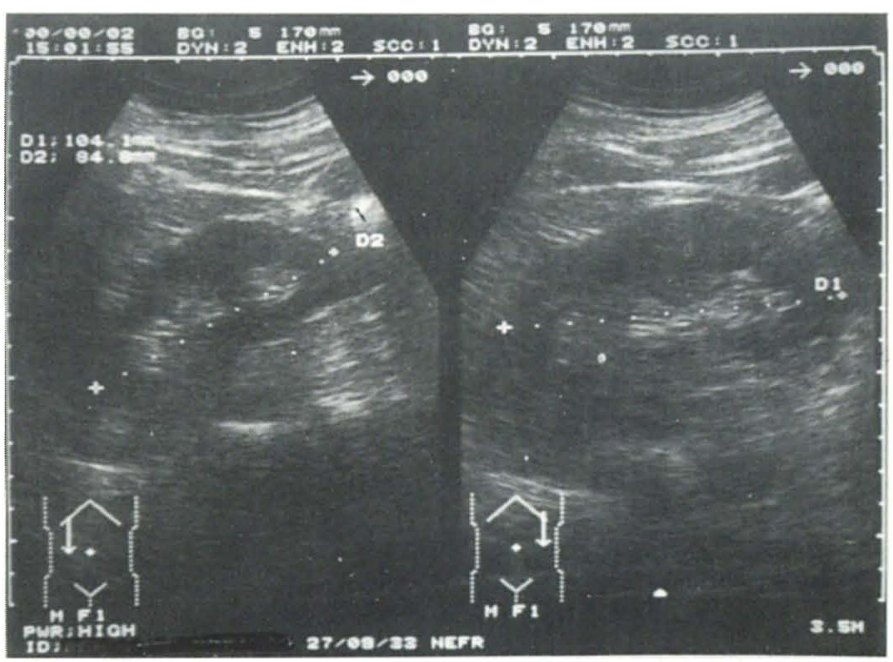

Figura 25: Malattia cistica acquisita del dializzato (ACKD); la regione corticale è invasa da numerosissime immagini cistiche in un paziente sottoposto da 9 anni a trattamento dialitico extracorporeo. 


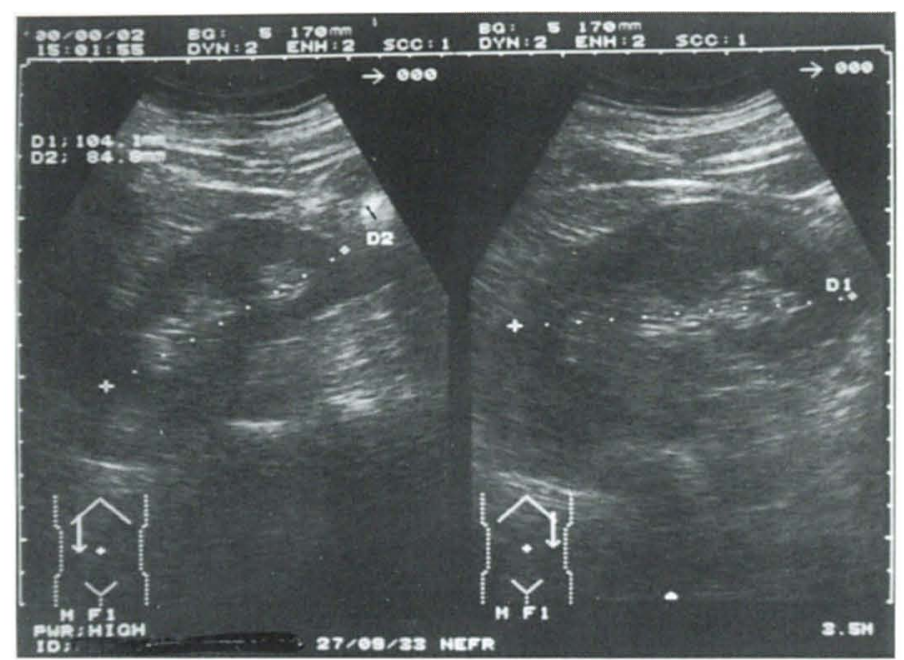

Figura 26: Ipertensione arteriosa; paziente con asimmetria renale: il rene ds molto più piccolo del controlaterale mostra un' ecostrottura armonica.

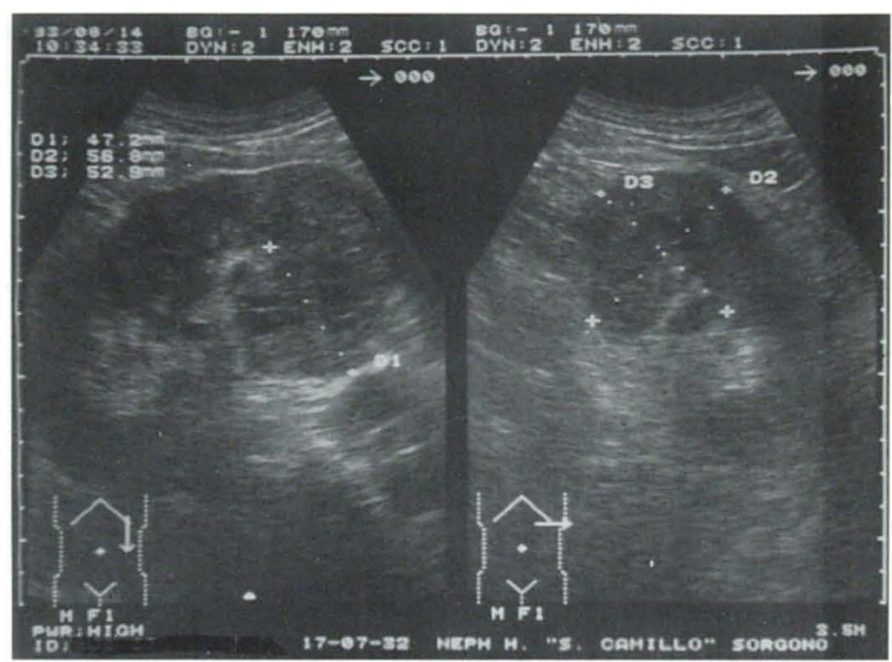

Figura 27: Massa neoplastica localizzata nella metà inferiore del rene sn. Il paziente era giunto alla nostra osservazione per una colica renale sn ed ematuria macroscopica. gine variamente ecogena. Un'immagine simile a quest'ultima si potrà avere anche nell'invasione del lume della vena renale da diffusione metastatica di un adenocarcinoma renale.

Parlando di studio ecografico renale, non possiamo non fare cenno alle masse solide (15), certamente un argomento che non rientra tra le nefropatie mediche, ma che dobbiamo ricordare perchè sono reperto occasionale, ma sicuramente non raro, nell'ambulatorio di ecografia nefrologica (fig. 27). Le masse tumorali sono generalmente rotondeggianti, spesso si può rilevare una immagine capsulare, a contenuto ecogeno di varia intensità con aree ipoecogene all'interno, segno di necrosi e di colliquazione. L'orientamento diagnostico è spesso facile di fronte a grosse masse tumorali, mentre è più complesso quando il tumore ha modeste dimensioni, per cui si pone la necessità di diagnosi differenziale con la pielonefrite batterica acuta, l'infarto renale, l'ematoma e, talora, l'ascesso renale.

\section{BIBLIOGRAFIA}

1. Weill F, Bihr E, et al. L'ultrasonographie rènal. Vigot, Paris, $2^{\mathrm{a}}$ ed., 1985.

2. Matter D. Echographie de l'appareil urinaire. Masson, Paris, 1986.

3. Rosenberg ER. Ultrasonographic evaluation of the kidney. Crit Rev Diagn Imaging, 1982; 17: $239-76$.

4. Capotondo L, Di Paolo N, et al. L'ecotomografia in nefrologia. Tecniche Nefrologiche \& Dialitiche, Wichtig Ed, 1990: 79-95.

5. Rosenfield AT, Siegel NJ. Renal parenchymal disease: histopatologic-sonographic correlation. Am J Rad, 1981; 138: 55.

6. Subramanyam BR. Renal Amyloidosis in juvenile rheumatoid arthritis: sonographic evaluation. Am J Rad, 1981; 138: 411.

7. Gilbert TG, Castellino RA. Linfomi maligni dell'apparato genitourinario. Dalla Palma, Progressi in Radiologia. Radiologia Lint Trieste, 1985.

8. Conti P, Mura C, et al. Eco- grafia nella calcolosi renale. Atti del II $^{\circ}$ Meeting di studio sull'ecotomografia in nefrologia. Milano 1992: in press.

9. Ravelli M. La malattia cistica renale all'ecotomografia. Giorn Tecn Nefrol Dial, 1992; 4, 2: 31-49.

10. Luscher TF, Wanner C, et al. Simple renal cyst and hypertension: cause or coincidence?. Clin Nephrol, 1986; 26: 19-24.

11. Cagliotti A, Armeli C, et al. Occurrence, signs and symptoms of simple renal cysts; preliminary results of a multicenter study. Seminar on Cystic Renal Disease, Wichtig Ed, 1989: 89-90.

12. Conti P, Mura C, et al. Ambulatorio di ecografia nefrologica: patologia renale cistica. Giorn It Nefr, 1993; 10, S4: 58.

13. Manns RA, Burrows FGO, et al. Acquired cystic disease of kidney: ultrasound as the primary procedure. Clin Rad, 1990; 41: 248-9.

14. Dazzi F. L'eco-doppler renale. Giorn Tecn Nefrol Dial, 1992; 4, 2 : 51-64.

15. Zanazzi M, Cavallotti $\mathrm{P}$, et al. Le masse solide renali all'ecotomografia. Giorn Tecn Nefrol Dial, 1992; 4, 2: 65-73. 